\title{
The double-layered chemical structure in DB white dwarfs
}

\author{
L. G. Althaus ${ }^{\star}$ and A. H. Córsico ${ }^{\star \star}$ \\ ${ }^{1}$ Facultad de Ciencias Astronómicas y Geofísicas, Universidad Nacional de La Plata, Paseo del Bosque S/N, \\ 1900 La Plata, Argentina \\ 2 Instituto de Astrofísica La Plata, IALP, CONICET \\ e-mail: acorsico@fcaglp.unlp.edu.ar
}

Received 29 August 2003 / Accepted 14 January 2004

\begin{abstract}
The purpose of this work is to study the structure and evolution of white dwarf stars with helium-rich atmospheres (DB) in a self-consistent way with the predictions of time-dependent element diffusion. Specifically, we have considered white dwarf models with stellar masses in the range $0.60-0.85 M_{\odot}$ and helium envelopes with masses from $10^{-2}$ to $10^{-4} M_{*}$. Our treatment of diffusion, appropriate for multicomponent gases, includes gravitational settling and chemical and thermal diffusion. OPAL radiative opacities for arbitrary metallicity and carbon-and oxygen-rich compositions are employed. Emphasis is placed on the evolution of the diffusion-modeled double-layered chemical structure. This structure, which is characterized by a pure helium envelope atop an intermediate remnant shell rich in helium, carbon and oxygen, is expected for pulsating DB white dwarfs, assuming that they are descendants of hydrogen-deficient PG 1159 post-AGB stars. We find that, depending on the stellar mass, if DB white dwarf progenitors are formed with a helium content smaller than $\approx 10^{-3} M_{*}$, a single-layered configuration is expected to emerge during the DB pulsation instability strip. We also explore the consequences of diffusively evolving chemical stratifications on the adiabatic pulsational properties of our DB white dwarf models. In this context, we find that the evolving shape of the chemical profile translates into a distinct behaviour of the theoretical period distribution as compared with the case in which the shape of the profile is assumed to be fixed during the evolution across the instability strip. In particular, we find that the presence of a double-layered structure causes the period spacing diagrams to exhibit mode-trapping substructures. Finally, we extend the scope of the calculations to the domain of the helium-rich carbon-contaminated DQ white dwarfs. In particular, we speculate that DQ white dwarfs with low detected carbon abundances would not be descendants of the PG 1159 stars.
\end{abstract}

Key words. stars: evolution - stars: interiors - stars: white dwarfs - stars: oscillations

\section{Introduction}

White dwarf stars with helium-rich atmospheres (DB) constitute about the $20 \%$ of all observed white dwarfs. The majority of these stars are thought to be the result of a very late thermal pulse experienced by post-asymptotic-giant-branch (post-AGB) progenitors during their early cooling phase (the born-again scenario; see Iben et al. 1983). As a result of such a pulse, most of the residual hydrogen envelope is completely burnt, and the star returns to the red giant region and then to the planetary nebula regime at high effective temperature ( $\left.T_{\text {eff }}\right)$ as a helium-burning object. Evolutionary calculations which incorporate diffusive overshooting (Herwig et al. 1999) show that the occurrence of a very late thermal pulse leads to the

Send offprint requests to: L. G. Althaus,

e-mail: althaus@fcaglp.unlp.edu.ar

* Member of the Carrera del Investigador Científico y Tecnológico, CONICET, Argentina.

$\star \star$ Fellow of the Consejo Nacional de Investigaciones Científicas y Técnicas (CONICET), Argentina. formation of hydrogen-deficient post-AGB stars with surface abundances of helium, carbon and oxygen. This prediction is in good agreement with the surface abundance pattern observed in most PG 1159 stars or the WR-type central stars of planetary nebulae (Dreizler \& Heber 1998), the hot and hydrogendeficient remnants of post-AGB stars generally believed to be the immediate progenitors of most DB white dwarfs.

The detection of pulsations in some DB white dwarfs (in the temperature range of $\approx 27000-20000 \mathrm{~K}$ ) has allowed researchers to provide independent valuable constraints on the internal structure and evolution of these stars, as well as on their progenitors. Indeed, asteroseismological techniques have become a powerful tool for inferring fundamental properties such the white dwarf mass, effective temperature, core composition and helium layer mass (see Bradley \& Winget 1994; Metcalfe et al. 2000; Metcalfe et al. 2001). In particular, Metcalfe et al. (2001) (see also Metcalfe et al. 2002) have recently applied DB white dwarf asteroseismology to place constraints on the ${ }^{12} \mathrm{C}(\alpha, \gamma){ }^{16} \mathrm{O}$ reaction rate from inferences for the abundance of central oxygen in the pulsating DB GD 358. It is worth 
noting that such studies are based on stellar models for pulsating DBs with a pure helium envelope atop the carbon-oxygen core (single-layered envelope).

However, recent theoretical evidence makes past inferences about core composition of DBs and the ${ }^{12} \mathrm{C}(\alpha, \gamma){ }^{16} \mathrm{O}$ reaction rate somewhat uncertain. Indeed, Fontaine \& Brassard (2002) have shown that the diffusion-built-up, double-layered chemical structure expected for most of the pulsating DBs leads to a distinct theoretical period distribution from that predicted by stellar models in which a single-layered configuration is assumed. The presence of a double-layered envelope in DB white dwarfs has been found in evolutionary calculations including time-dependent element diffusion by Dehner \& Kawaler (1995) and Gautschy \& Althaus (2002), assuming that DB white dwarfs descend from PG 1159 stars. Indeed, such calculations show that by the time the domain of the variable DBs is reached, models are characterized by two different chemical transition zones (double-layered configuration). In fact, above the carbon-oxygen core, there exists an envelope consisting of an intershell region rich in helium, carbon and oxygen, the relics of the short-lived mixing episode occurred during the last helium thermal pulse, and an overlying pure helium mantle resulting from gravitational settling of carbon and oxygen. Finally, DB asteroseismological fittings incorporating both the double-layered envelope feature expected from time-dependent diffusion calculations and adjustable carbon-oxygen cores have recently been presented by Metcalfe et al. (2003) for a wide range of helium contents and stellar masses. Despite their models yielding significantly better fits to observations, the derived stellar parameters for some fittings led them to conclude that double-layered models with adjustable carbon/oxygen cores may not be entirely appropriate to explain the observations.

In view of these concerns, this paper is aimed at exploring the evolution of DB white dwarfs in a self-consistent way with the predictions of time-dependent element diffusion. Emphasis is placed on the chemistry variations along the evolutionary stages during which these stars are expected to pulsate. Specifically, we hope to address the following question: is it possible that the diffusion-induced, double-layered structure could be altered by further chemical evolution to such an extent that a single-layered configuration emerges before the star reaches the red edge of the instability strip? In view of the lack of previous calculations of the mass-dependence of timedependent diffusion profiles, we judge that this question is an important one to be answered. Additionally, we extend the scope of the paper and explore the consequences of diffusively evolving chemical stratifications for the adiabatic pulsational properties of the models. Finally, the implications of our results for the carbon surface abundance in some helium-rich DQ white dwarfs are explored.

\section{Computational details and input physics}

The results presented in this work have been obtained with the DB white dwarf evolutionary code developed at La Plata Observatory. Except for minor modifications, the code is basically that described in Gautschy \& Althaus (2002) and references cited therein. In particular, microphysics includes an updated version of the equation of state of Magni \& Mazzitelli (1979), OPAL radiative opacities for arbitrary metallicity (Iglesias \& Rogers 1996) including carbon- and oxygenrich compositions, and up-to-date neutrino emission rates and conductive opacities (see Althaus et al. 2002). In particular, opacities for various metallicities are required because of the metallicity gradient that develops in the envelopes as a result of gravitational settling and also in the outer layers of DQ white dwarfs because of dredge-up episodes (see next section). In this work, convection is treated in the framework of the mixing length theory as given by the ML2 parametrization (see Tassoul et al. 1990).

The evolution of the chemical abundance distribution caused by diffusion processes has been fully accounted for by means of a time-dependent, finite-difference scheme that solves elemental continuity equations. To compute the white dwarf evolution in a self-consistent way with the predictions of element diffusion, such a scheme is coupled to the evolutionary code. In our treatment of time-dependent diffusion we have considered gravitational settling and chemical and thermal diffusion for the nuclear species ${ }^{4} \mathrm{He},{ }^{12} \mathrm{C}$ and ${ }^{16} \mathrm{O}$. Radiative levitation, which is important in connection with surface composition of hot white dwarfs has been neglected. Specifically, we have adopted the treatment of Burgers (1969) for multicomponent gases. There, diffusion velocities $w_{i}$ and residual heat flows $r_{i}$ satisfy the set of equations

$$
\begin{aligned}
\frac{\mathrm{d} p_{i}}{\mathrm{~d} r}-\frac{\rho_{i}}{\rho} \frac{\mathrm{d} p}{\mathrm{~d} r}-n_{i} Z_{i} \mathrm{e} E= & \sum_{j \neq i}^{N} K_{i j}\left(w_{j}-w_{i}\right) \\
& +\sum_{j \neq i}^{N} K_{i j} z_{i j} \frac{m_{j} r_{i}-m_{i} r_{j}}{m_{i}+m_{j}}
\end{aligned}
$$

and

$$
\begin{aligned}
\frac{5}{2} n_{i} k_{\mathrm{B}} \nabla T= & -\frac{5}{2} \sum_{j \neq i}^{N} K_{i j} z_{i j} \frac{m_{j}}{m_{i}+m_{j}}\left(w_{j}-w_{i}\right)-\frac{2}{5} K_{i i} z_{i i}{ }^{\prime \prime} r_{i} \\
& -\sum_{j \neq i}^{N} \frac{K_{i j}}{\left(m_{i}+m_{j}\right)^{2}}\left(3 m_{i}^{2}+m_{j}^{2} z_{i j}{ }^{\prime}+0.8 m_{i} m_{j} z_{i j}{ }^{\prime \prime}\right) r_{i} \\
& +\sum_{j \neq i}^{N} \frac{K_{i j} m_{i} m_{j}}{\left(m_{i}+m_{j}\right)^{2}}\left(3+z_{i j}{ }^{\prime}-0.8 z_{i j}{ }^{\prime \prime}\right) r_{j} .
\end{aligned}
$$

Here, $p_{i}, \rho_{i}, n_{i}, Z_{i}$ and $m_{i}$ means, respectively, the partial pressure, mass density, number density, mean charge and mass for species $i$ ( $N$ means the number of ionic species plus electron). $T, k_{\mathrm{B}}$ and $\nabla T$ are the temperature, Boltzmann constant and temperature gradient, respectively. The unknown variables are $w_{i}$ and $r_{i}$ (for ions and electrons). The electric field $E$ has also to be determined. The resistance coefficients $\left(K_{i j}, z_{i j}, z_{i j}{ }^{\prime}\right.$ and $\left.z_{i j}{ }^{\prime \prime}\right)$ are from Paquette et al. (1986). For more details, see Gautschy \& Althaus (2002). It is worth mentioning that a recent study of the ${ }^{3} \mathrm{He}$ diffusion in DB white dwarfs has been presented by Montgomery et al. (2001).

The shape of the chemical profile in the envelope of a white dwarf is determined by the competition between basically partial pressure gradients, gravity and induced electric fields. 


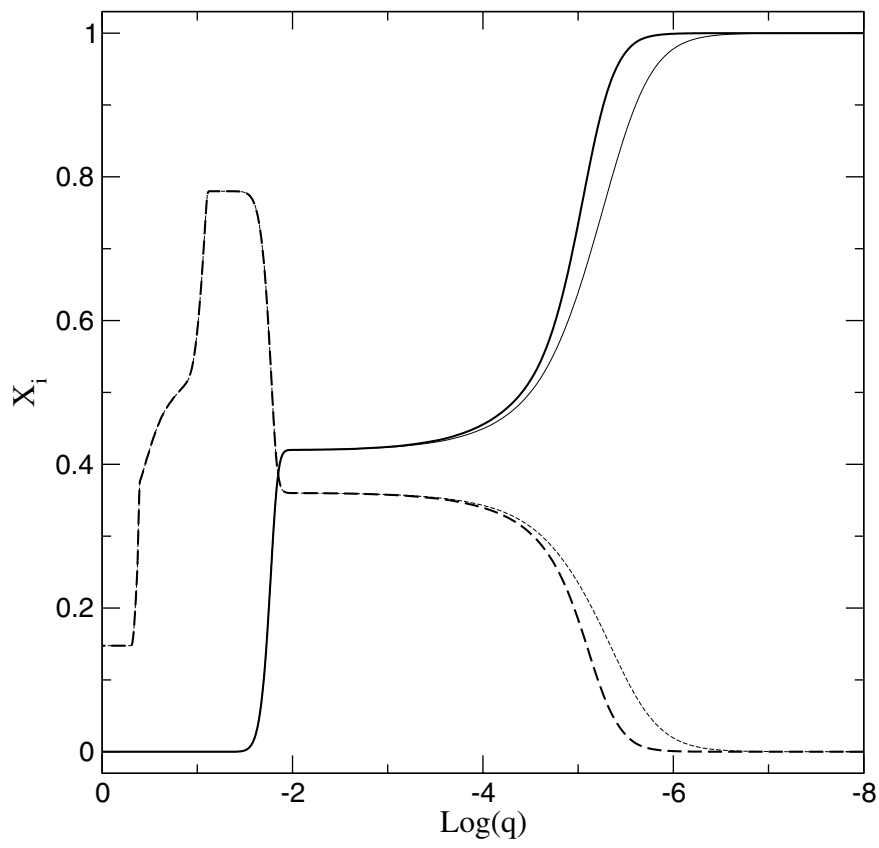

Fig. 1. Abundance by mass of ${ }^{4} \mathrm{He}$ and ${ }^{12} \mathrm{C}$ (solid and dashed line, respectively) as a function of the outer mass fraction $q$ for $0.60-M_{\odot}$ DB models at $T_{\text {eff }}=27300 \mathrm{~K}$. Thick (thin) lines correspond to the situation in which thermal diffusion is considered (neglected). Clearly, the inclusion of thermal diffusion leads to thicker pure helium mantles.

In order to get an insight into the essential physics driving element diffusion and to understand some of the results to be presented later we will assume for a moment a simplified situation in which only two ionic species with mean charge $Z_{1}$ and $Z_{2}$ and atomic mass number $A_{1}$ and $A_{2}$ are present. In addition, we assume the electron to have zero mass and neglect the contribution from thermal diffusion. It is then straightforward to show from Eq. (1) that the diffusion velocity satisfies

$$
\begin{aligned}
K_{12} w_{1} f= & \left(\frac{A_{2}}{Z_{2}}-\frac{A_{1}}{Z_{1}}\right) m_{\mathrm{H}} g \\
& +\left(\frac{1}{Z_{2}}-\frac{1}{Z_{1}}\right) k_{\mathrm{B}} T \frac{\mathrm{d} \ln T}{\mathrm{~d} r}+\frac{k_{\mathrm{B}} T}{Z_{2} n_{2}} \frac{\mathrm{d} n_{2}}{\mathrm{~d} r}-\frac{k_{\mathrm{B}} T}{Z_{1} n_{1}} \frac{\mathrm{d} n_{1}}{\mathrm{~d} r},
\end{aligned}
$$

where $w_{1}$ is the diffusion velocity for specie $1\left(w_{1}<0\right.$ means that the element sinks into the star) and $f=(1+$ $\left.A_{1} n_{1} / A_{2} n_{2}\right)\left(n_{1} Z_{1}+n_{2} Z_{2}\right) / n_{1} Z_{1} n_{2} Z_{2}$. In Eq. (3), $m_{\mathrm{H}}$ and $g$ are the hydrogen-atom mass and gravity, respectively. In deriving Eq. (3) we have assumed an ideal gas for ions and use the condition for no net mass flow relative to the centre of mass. The first term on the right-hand side of Eq. (3) takes into account the contribution of the gravitational settling and the influence of the induced electric field, and the third and fourth terms refer to the chemical diffusion contribution. We apply Eq. (3) to a mixture of ${ }^{4} \mathrm{He}$ and ${ }^{12} \mathrm{C}$. Note that the ionization state of elements strongly affects the diffusion velocity. In particular, in the nondegenerate outer layers, ${ }^{12} \mathrm{C}$ is not fully ionized (for instance, in our $T_{\text {eff }}=27300 \mathrm{~K}, 0.6-M_{\odot}$ model, ${ }^{12} \mathrm{C}$ becomes completely ionized at a mass depth of $10^{-8} M_{*}$ below the stellar surface), and as a result ${ }^{4} \mathrm{He}$ diffuses upwards very rapidly driven mainly by gravity (first term in Eq. (3) is dominant). As cooling proceeds, this leads to a thickening pure helium mantle. In deeper, more degenerate layers, gravitational settling turns out to be less operative. Indeed, ${ }^{12} \mathrm{C}$ (and ${ }^{4} \mathrm{He}$ ) becomes fully ionized and the gravity term vanishes. There, diffusion is driven essentially by chemical gradients, thus forcing helium to penetrate downwards to hotter layers. Note that this is quite different from the situation for a mixture of ${ }^{1} \mathrm{H}$ and ${ }^{4} \mathrm{He}$. In that case, the gravity term never vanishes and, at large degeneracy, it takes over chemical diffusion, thus halting the inward diffusion of ${ }^{1} \mathrm{H}$ and leading to a complete separation of the two species at advanced ages.

It is important to note that our diffusion treatment considers thermal diffusion. This is by no means a negligible contribution to the diffusion processes. Because thermal diffusion acts in the same direction as gravitational settling, the neglect of thermal diffusion underestimates the rate at which carbon and oxygen diffuse downwards. Hence, at a given $T_{\text {eff }}$ value, we expect a thicker pure helium mantle when thermal diffusion is taken into account. This expectation is indeed borne out by Fig. 1 which illustrates the helium and carbon abundance distribution in terms of the outer mass fraction $q\left(q=1-M_{r} / M_{*}\right)$ for a $0.6-M_{\odot} \mathrm{DB}$ white dwarf model at $T_{\text {eff }}=27300 \mathrm{~K}$, i.e. near the blue edge of the DB instability strip. Note that the mass embraced by the pure helium mantle amounts to $2.5 \times 10^{-6} M_{*}$, as compared with the value of $6 \times 10^{-7} M_{*}$ when thermal diffusion is neglected.

With regard to the initial stellar models needed to start our cooling sequences, they were obtained by means of the same artificial procedure as described in Gautschy \& Althaus (2002). In particular, such starting models correspond to hydrogendeficient post-AGB configurations with an uniform envelope chemical composition of helium, carbon and oxygen (with abundances of $0.42,0.36$ and 0.22 by mass, respectively). As mentioned in the introduction, the envelope composition is the result of a short-lived mixing event occurred during the late thermal pulse experienced by a post-AGB remnant. The chemical composition of the core is that of Salaris et al. (1997). Specifically, we have considered DB white dwarf models with stellar masses of $0.60,0.65,0.70,0.75$ and $0.85 M_{\odot}$. The mass of the helium envelope $\left(M_{\mathrm{He}}\right)$ is constrained by the theory of post-AGB evolution to be in the range $10^{-2}$ to $10^{-3} M_{*}$, in good agreement with carbon dredge-up simulations in DQ white dwarfs, the cooler descendants of DBs, by MacDonald et al. (1998) for a typical white dwarf mass of $0.6 M_{\odot}$. In this work, we considered the following values for $M_{\mathrm{He}}: 8 \times 10^{-3}, 9 \times 10^{-4}$ and $10^{-4} M_{*}$.

In what follows, we describe the main results of our calculations. We want to mention that this paper is not aimed at performing asteroseismological fittings to a particular object. Rather, we concentrate mainly on the consequences of element diffusion for the chemical profiles expected at the DB instability strip. With regard to pulsational properties, our attention is directed exclusively towards the effects of diffusively evolving chemical profiles on the adiabatic pulsational properties of the DB white dwarf models. In this sense, a detailed description of the evolutionary phases prior to the formation of DB white dwarfs, particularly regarding the chemical profile for the carbon-oxygen core, is not of primary importance for our purposes. As for pulsational calculations, we have 


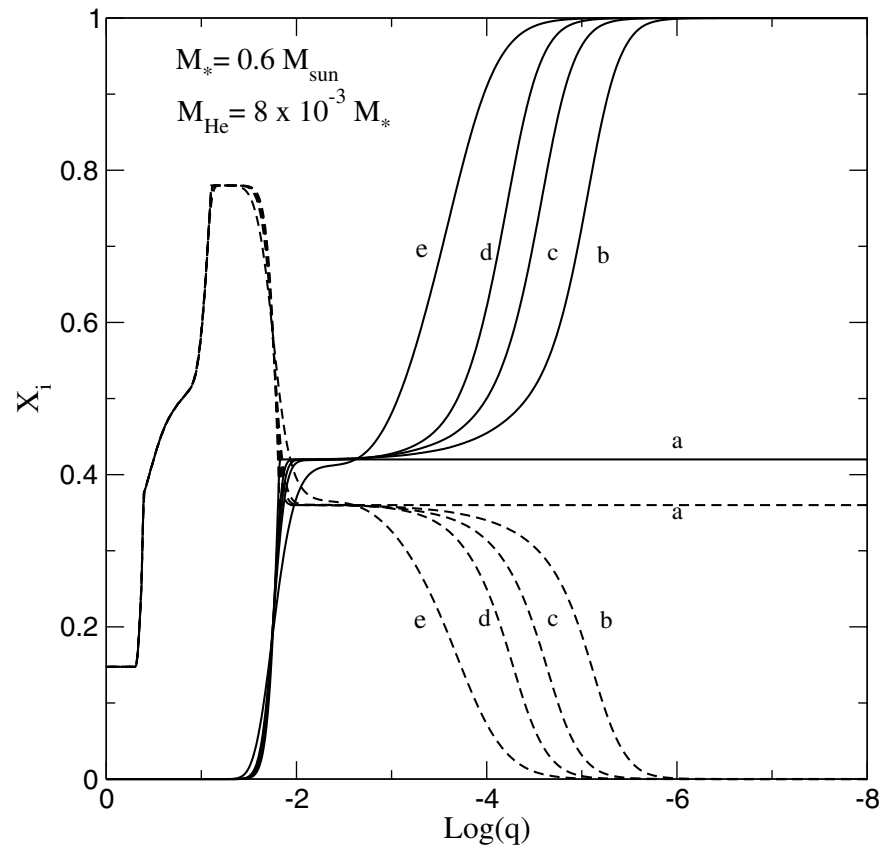

Fig. 2. Evolution of the abundance by mass of ${ }^{4} \mathrm{He}$ (solid lines) and ${ }^{12} \mathrm{C}$ (dashed lines) as a function of the outer mass fraction $(q)$ for $0.60-M_{\odot}$ DB models having a helium content of $M_{\mathrm{He}}=8 \times$ $10^{-3} M_{*}$. Starting from a model with an initially homogeneous envelope (curves a), following models (b, c, d and e) correspond to evolutionary stages characterized by $T_{\text {eff }}=27500,23000,20200$ and $10200 \mathrm{~K}$. Gravitationally induced diffusion leads to the development of a double-layered structure. Note also that the outer layer abundance distribution evolves appreciably as the white dwarf cools down through the stages where DBs are found to pulsate.

computed adiabatic, nonradial $g$-modes of the DB white dwarf models with the pulsational code employed in Córsico et al. (2001), appropriately modified to study pulsating DB white dwarfs. In particular, the treatment we follow to assess the Brunt-Väisälä frequency $(N)$ is that proposed by Brassard et al. (1991).

\section{Evolutionary and pulsational results}

We begin by examining Fig. 2 which illustrates the evolution of the internal ${ }^{4} \mathrm{He}$ and ${ }^{12} \mathrm{C}$ distribution as a function of the outer mass fraction $q$ for $0.60-M_{\odot}$ DB white dwarf models with a helium content of $8 \times 10^{-3} M_{*}$. In particular, the expectation at three selected values of $T_{\text {eff }}$ that cover the temperature range for pulsating DB white dwarfs is depicted. Clearly, diffusion processes appreciably modify the chemical profiles as the white dwarf evolves, making essentially the bulk of helium float to the surface and heavier element sink out of surface layers. Note that, as found in previous studies (Dehner \& Kawaler 1995; Gautschy \& Althaus 2002; Fontaine \& Brassard 2002) the formation of a double-layered structure is expected by the time the white dwarf has reached the domain of pulsational instability. Indeed, diffusion proceeds efficiently, giving rise to pure helium outermost layers. Specifically, we find that at $T_{\text {eff }} \approx 27500 \mathrm{~K}$, the $0.6-M_{\odot}$ DB model is characterized by a pure helium mantle of $2 \times 10^{-6} M_{*}$ (curve b) and an underlying intershell (still-uniform) region rich in helium, carbon and oxygen. We note that the pure helium mantles of our models are somewhat larger than those reported by Dehner \& Kawaler (1995) and Fontaine \& Brassard (2002). In part, the difference is due to the inclusion of thermal diffusion in our simulations (see Sect. 2). Interestingly, the chemical profile evolves appreciably with the further evolution through the DB instability strip, as shown by Fig. 2. It is worth noting that, during such stages, element diffusion not only thickens the pure helium mantle but also it modifies the shape of the chemical profile, a feature which is expected to leave some traces in the theoretical period distribution, as compared with the case in which the shape of the profile is assumed to be fixed during the DB white dwarf evolution across the instability strip (see later in this section).

Although the attention of this paper is focused on the evolutionary stage corresponding to variable DBs, we have followed the further evolution of some sequences down to the domain of the carbon-enriched DQ white dwarfs. The resulting chemical profile for the case of a $0.6-M_{\odot}$ DB model with a helium content of $M_{\mathrm{He}}=8 \times 10^{-3} M_{*}$ at $T_{\mathrm{eff}}=10200 \mathrm{~K}$ is shown by curve e in Fig. 2. Note that even at such advanced stages, diffusion has not engulfed the entire helium-carbon-oxygen intershell, and the chemical profile is still characterized by a double-layered structure. In particular, the pure helium mantle extends down to a fractional mass depth $q \approx 5 \times 10^{-5} M_{*}$. Note that, despite the total helium content amounting to a fraction of $\approx 0.01$ of the stellar mass, there is a substantial amount of carbon stretching outwards from the intershell region. We expect then a significant carbon enrichment in the surface layers as a result of convective dredge-up of the carbon diffusive tail, a process which is generally believed to be responsible for the observed carbon in DQs. This expectation is indeed borne out by Fig. 3, which shows the carbon abundance distribution in terms of the outer mass fraction at various $T_{\text {eff }}$ values for the $0.60-M_{\odot}$ DB white dwarf sequence with a helium mass of $8 \times 10^{-3} M_{*}$. Chemical diffusion causes some carbon from deeper layers to diffuse upwards (see Eq. (3)) to where convection dredge it up to the surface. As cooling proceeds, the base of the convection zone moves inwards and eventually reaches a maximum depth of $4.2 \times 10^{-6} M_{\odot}$ by $T_{\text {eff }} \approx 10000 \mathrm{~K}$. The depth reached by the convection zone, which at this low effective temperature is almost independent of the treatment of convection, is limited by the low conductive opacity of the degenerate interior. It is worth noting that the presence of carbon (and dredged metals) in the outer layers renders the helium plasma less transparent, thus providing a not so deep convection zone. Our results suggest that at $T_{\text {eff }}=10000 \mathrm{~K}$, the carbon surface abundance reaches ${ }^{1} \log n\left({ }^{12} \mathrm{C}\right) / n\left({ }^{4} \mathrm{He}\right) \approx-4$. This abundance far exceeds the lowest carbon abundance observed in many DQs (see MacDonald et al. 1998). We speculate that DB models less massive than $0.6 M_{\odot}$ evolved directly from PG 1159

\footnotetext{
${ }^{1}$ In terms of a single-layered profile, a pure helium mantle of $\approx 5 \times$ $10^{-5} M_{*}$ corresponds to a total helium content of $10^{-3}-10^{-4} M_{*}$. Thus, our quoted surface carbon abundance is in rough comparable agreement with the prediction of MacDonald et al. (1998) for their singlelayered sequences with a helium content of about $10^{-3} M_{*}$.
} 


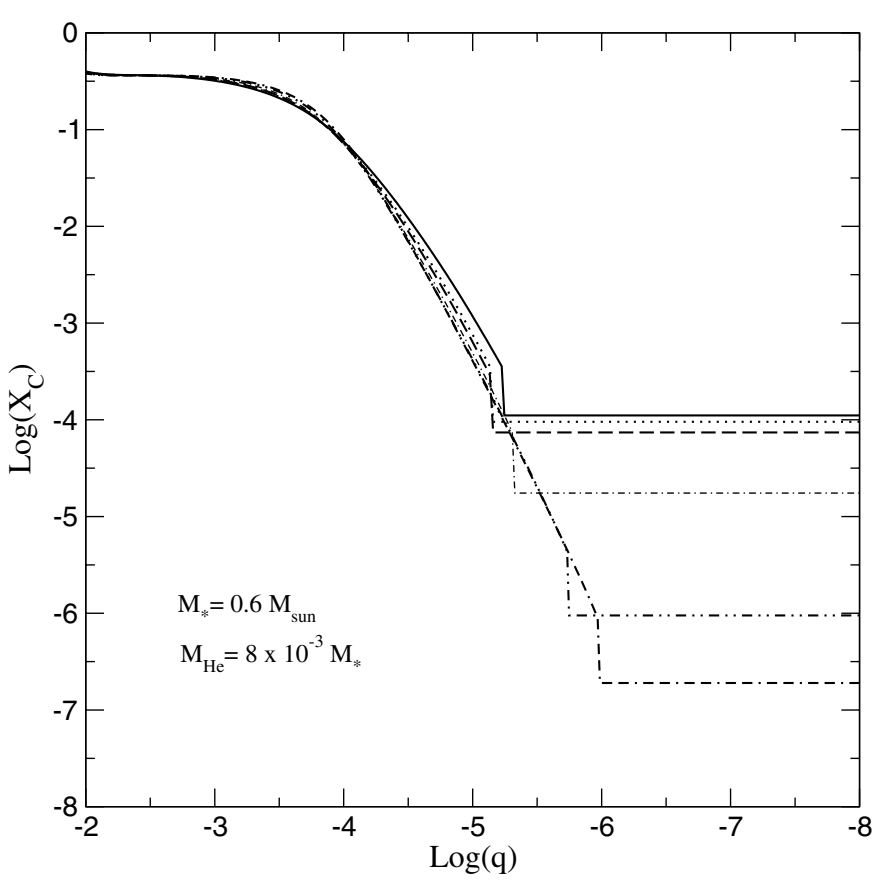

Fig. 3. Abundance distribution of ${ }^{12} \mathrm{C}$ in the outer layers at (from bottom to top) $T_{\text {eff }}=14000,13300,11900,10700,10200$ and $9200 \mathrm{~K}$. Models correspond to a $0.60-M_{\odot}$ DB white dwarf having a helium content of $M_{\mathrm{He}}=8 \times 10^{-3} M_{*}$. Note that, as a result of convection reaching the ${ }^{12} \mathrm{C}$ diffusive tail, the outer layers become substantially ${ }^{12} \mathrm{C}$-enriched.

configurations should also exhibit such large carbon surface abundances. In fact, even in this case, settling of carbon from the outer layers would take long time, and accordingly, a substantial amount of carbon would be dredged upwards by convection. Additional evolutionary calculations for such lowmass DB white dwarfs are required to place this statement on a solid background. Hence, the plausibility of an evolutionary link between low-mass PG 1159 and DQ stars with low detected carbon abundance is not clear if canonical convective dredge-up is the source of the observed carbon for such DQs.

In the case of small helium contents, the shape of the double-layered profile is strongly affected by element diffusion. This is illustrated by Figs. 4 and 5, which show the internal ${ }^{4} \mathrm{He}$ and ${ }^{12} \mathrm{C}$ distribution as a function of $q$ for $0.60-M_{\odot}$ DB white dwarf models with a helium mass of $9 \times 10^{-4} M_{*}$ and $1 \times 10^{-4} M_{*}$, respectively. On the basis of these figures, we conclude that the double-layered structure is altered by chemical evolution to such a degree that a single-layered configuration emerges even during the stages of pulsation instability. This result is not surprising in view of Eq. (3). Indeed, for small helium contents, electron degeneracy occurs below the helium-carbon-oxygen mantle, and as a result, the second term in Eq. (3) is dominant, leading to the formation of a single-layered structure in relatively short ages. Specifically, for $M_{\mathrm{He}}=9 \times 10^{-4} M_{*}$ a single-layered configuration occurs by the time the star reaches the end of the instability strip, and for $M_{\mathrm{He}}=1 \times 10^{-4} M_{*}$, a single-layered configuration sets in even by the onset of pulsation instability. Hence, if post-AGB, DB white dwarf progenitors are formed with a helium

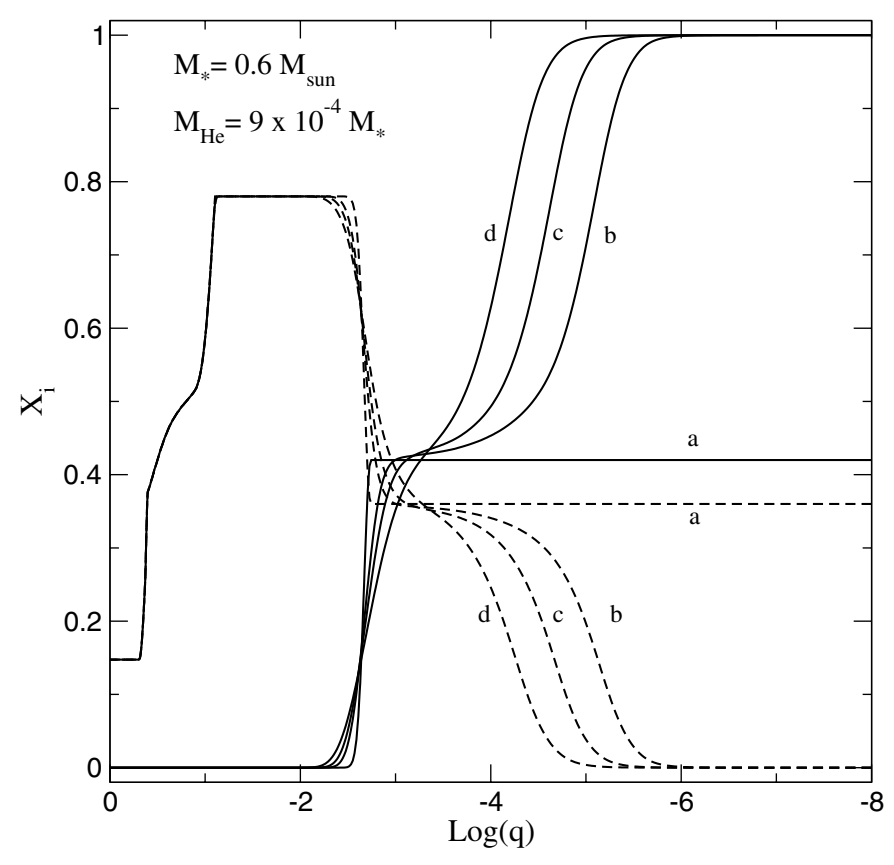

Fig. 4. Same as Fig. 2 but for a helium content of $M_{\mathrm{He}}=9 \times$ $10^{-4} M_{*}$. Starting from a model with an initially homogeneous envelope (curves a), following models (b, c and d) correspond to evolutionary stages characterized by $T_{\text {eff }}=27200,22900$ and $19800 \mathrm{~K}$. Here, the shape of the chemical profile is notably modified by diffusion processes. In particular, shortly after the red edge of the instability strip is reached, the double-layered structure has virtually disappeared (curves d).

content smaller than $\approx 10^{-3} M_{*}$, the double-layered structure is expected to become single-layered by the time the star reaches the red edge of the DB instability strip.

The dependence of the chemical profile evolution upon the stellar mass is shown in Figs. 6 to 8. In particular, in Fig. 6 we compare the evolution of the chemical profile for $0.75-M_{\odot} \mathrm{DB}$ models with the same helium content than that considered in Fig. $4\left(M_{\mathrm{He}}=9 \times 10^{-4} M_{*}\right)$. The role of diffusion in relatively massive DB white dwarfs is clearly documented in this figure. Indeed, here, over most of the temperature range of DB variables, the chemical profile bears no signature of a double-layered structure. By contrast, in the case of 0.6- $M_{\odot}$ models the double-layered profile turns into a singlelayered one only after evolution has proceeded to effective temperatures somewhat below the red edge of the instability strip. In Figs. 7 and 8 we compare the shape of the helium profile for various stellar masses at a fixed $T_{\text {eff }}$. Note that the shape of chemical profile is very dependent on the stellar mass of the star.

Next, we examine the pulsational properties of our models. We shall consider non-radial $(\ell=1) g$-modes with periods $P_{k}$ ( $k$ being the radial overtone) in the range of 100-1000 s. Figures 9-11 display the forward period spacing $\left(\Delta P_{k} \equiv P_{k+1}-\right.$ $P_{k}$ ) as a function of period (period spacing diagrams) for the case of 0.6- $M_{\odot}$ DB white dwarf models with helium contents of $M_{\mathrm{He}}=8 \times 10^{-3} M_{*}, 9 \times 10^{-4} M_{*}$ and $1 \times 10^{-4} M_{*}$, respectively. In each figure results corresponding to a sequence of four selected models at decreasing effective temperatures (as labeled in left 


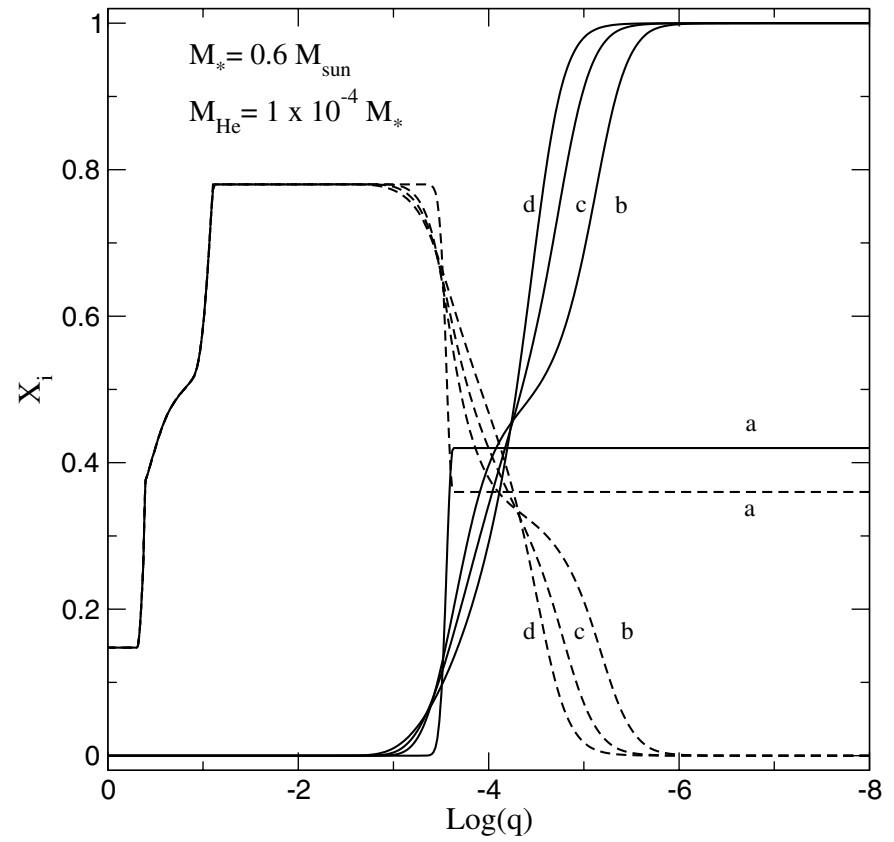

Fig. 5. Same as Fig. 2 but for a helium content of $M_{\mathrm{He}}=1 \times 10^{-4} M_{*}$. Starting from a model with an initially homogeneous envelope (curves a), following models ( $b, c$ and d) correspond to evolutionary stages characterized by $T_{\text {eff }}=27300,23600$ and $20600 \mathrm{~K}$. Here, the chemical profile bears no clear signature of a double-layered structure even at the hottest $T_{\text {eff }}$ values considered (curves b). Models, instead, are characterized by a single-layered structure.

panels) covering the pulsational strip are depicted. The left column of panels (solid lines) depicts the $\Delta P_{k}$ distributions as predicted by calculations in which element diffusion has been accounted for, and the central column of panels (dashed lines) shows the same information but for the case in which diffusion processes have been locked in the evolutionary computations, e.g., the chemical profiles remain fixed with further evolution from a $T_{\text {eff }}$ value corresponding approximately to the blue edge of the DB instability strip. Finally, the right column of panels documents the differences between the values of $\Delta P_{k}$ as given by diffusively evolving profiles and the fixed ones.

An inspection of the figures indicates that in both sets of computations the pulsation mode spectrum changes markedly as the white dwarf models cool down to the red extreme of the DB instability strip. In the case of models with diffusion, such changes are caused by: (1) the changes the mechanical and thermal structure of models in response to energy looses, changes which are translated mainly into a decreasing in the Brunt-Väis̈alä frequency at the core region and the consequent increasing of periods and period spacings, and (2) the evolution of the chemical profiles caused by diffusive processes, thus giving rise to changes in the location of the well known Brunt-Väisälä frequency's bumps associated with chemical interfaces. Instead, in the case of models in which chemical diffusion is not considered, the changes in the period pattern are due only to the secular changes resulting from cooling (item 1), e.g., the Brunt-Väisälä frequency decreases at the innermost regions but the profile of the Ledoux term $B$ (a quantity closely involved in the computation of the Brunt-Väisälä frequency at

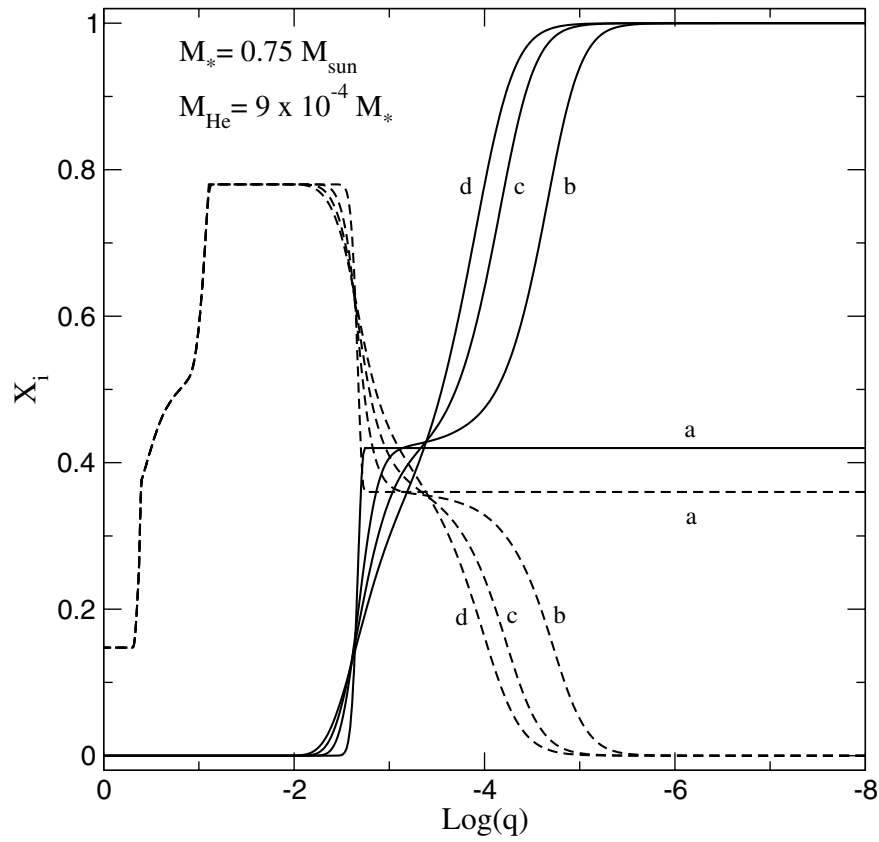

Fig. 6. Same as Fig. 4 but for a stellar mass of $0.75 M_{\odot}$. Starting from a model with an initially homogeneous envelope (curves a), following models ( $b, c$ and $d$ ) correspond to evolutionary stages characterized by $T_{\text {eff }}=27200,23100$ and $19900 \mathrm{~K}$. Note that, a single-layered profile emerges at larger $T_{\text {eff }}$ values as compared with the $0.6 M_{\odot}$ model.

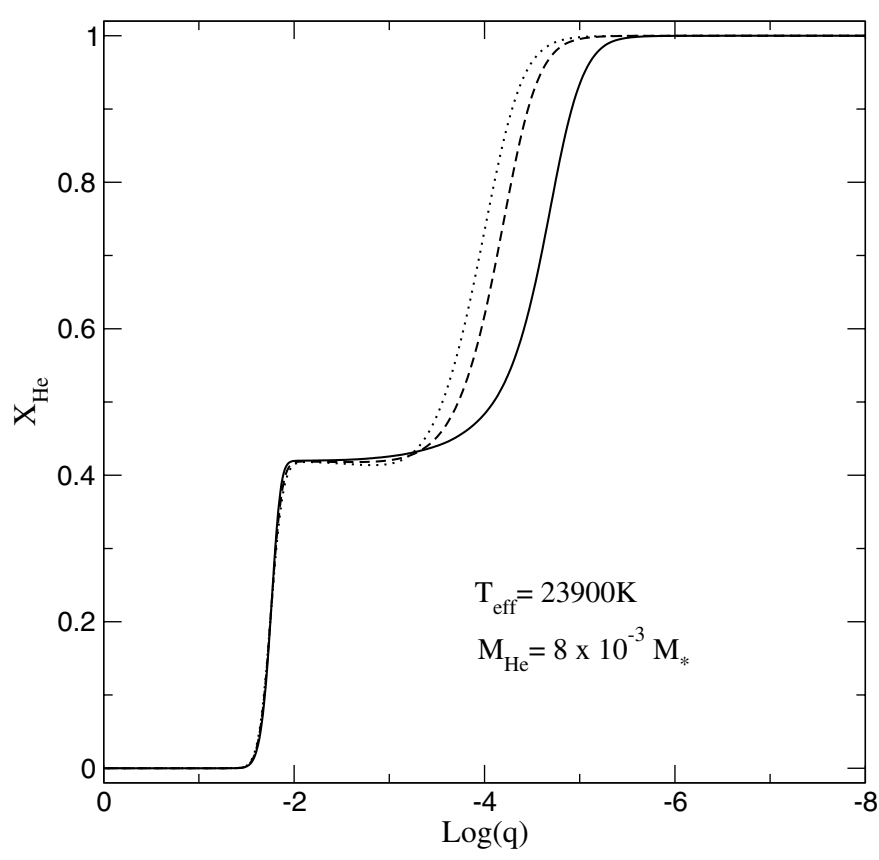

Fig. 7. Abundance by mass of ${ }^{4} \mathrm{He}$ as a function of the outer mass fraction $(q)$ for $0.60,0.75$ and $0.85-M_{\odot}$ DB models (solid, dashed and dotted lines, respectively), having a helium content of $M_{\mathrm{He}}=8 \times$ $10^{-3} M_{*}$. Models correspond to $T_{\text {eff }}=23900 \mathrm{~K}$.

the chemical interfaces; see Brassard et al. 1991 for details) remains almost fixed. Interestingly enough, during the instability strip the period spacing distribution is strongly modified according to whether element diffusion is considered or not. 


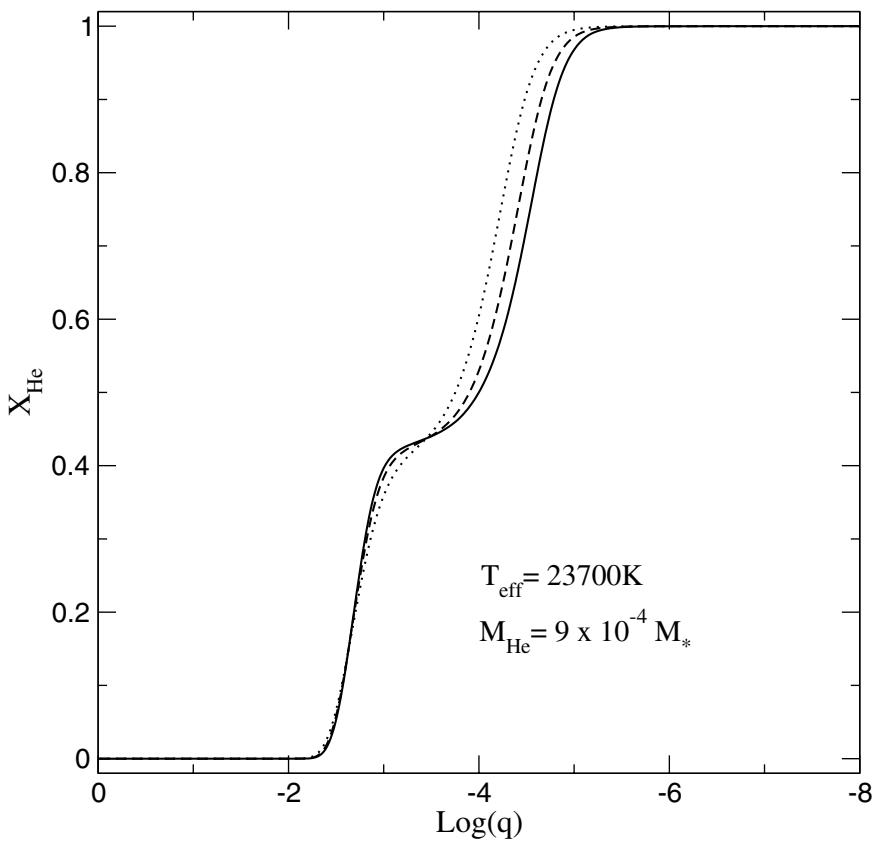

Fig. 8. Same as Fig. 7 but for a helium content of $M_{\mathrm{He}}=9 \times 10^{-4} M_{*}$. Solid, dashed and dotted lines correspond to DB models with 0.60 , 0.70 and $0.75-M_{\odot}$ at $T_{\text {eff }}=23700 \mathrm{~K}$.

It is interesting to note that the period spacing diagrams of the pulsation mode spectrum exhibits a clear mode-trapping substructure when a multi-layered structure is present, and more importantly how this substructure change due to diffusion. In fact, from left panels of Fig. 9 it can be seen that there is substructure at all evolutionary stages depicted, whereas in Fig. 10 the period spacing diagram becomes steadly simpler as the $T_{\text {eff }}$ decreases. Finally note that in Fig. 11 the period spacing diagram is characterized by a fairly clean structure at all temperatures shown. The occurrence of substructures in the period diagrams is of course intimately related to the presence or not of the double-layered structure, a fact that can be related directly to Figs. 2, 4 and 5. That is, the substructure is determined by whether or not the pure helium layer has had time to engulf the whole helium-carbon-oxygen intershell. In the case of a single-layered structure there is a single strong mode-trapping cycle, otherwise there are two cycles of differing strength.

Finally, the period spacing differences between model with and without diffusion (as they were defined before) are by no means negligible and, as can be visualized from the plots, amounts to $\approx \pm 8$ s for all the helium contents we have considered. These differences far exceed the quoted values of rms differences between the observed and calculated period spacings $\left(\sigma_{\Delta P}\right)$ in current detailed asteroseismological fits to pulsating DB white dwarf stars. In light of these results, we conclude that the dependence of the shape of the evolving chemical profiles on the effective temperature cannot be entirely ignored, that is, in order to maintain physical consistency, these parameters cannot be considered as independent ones when attempts at performing detailed seismological fit to a particular star are made. These parameters are actually not
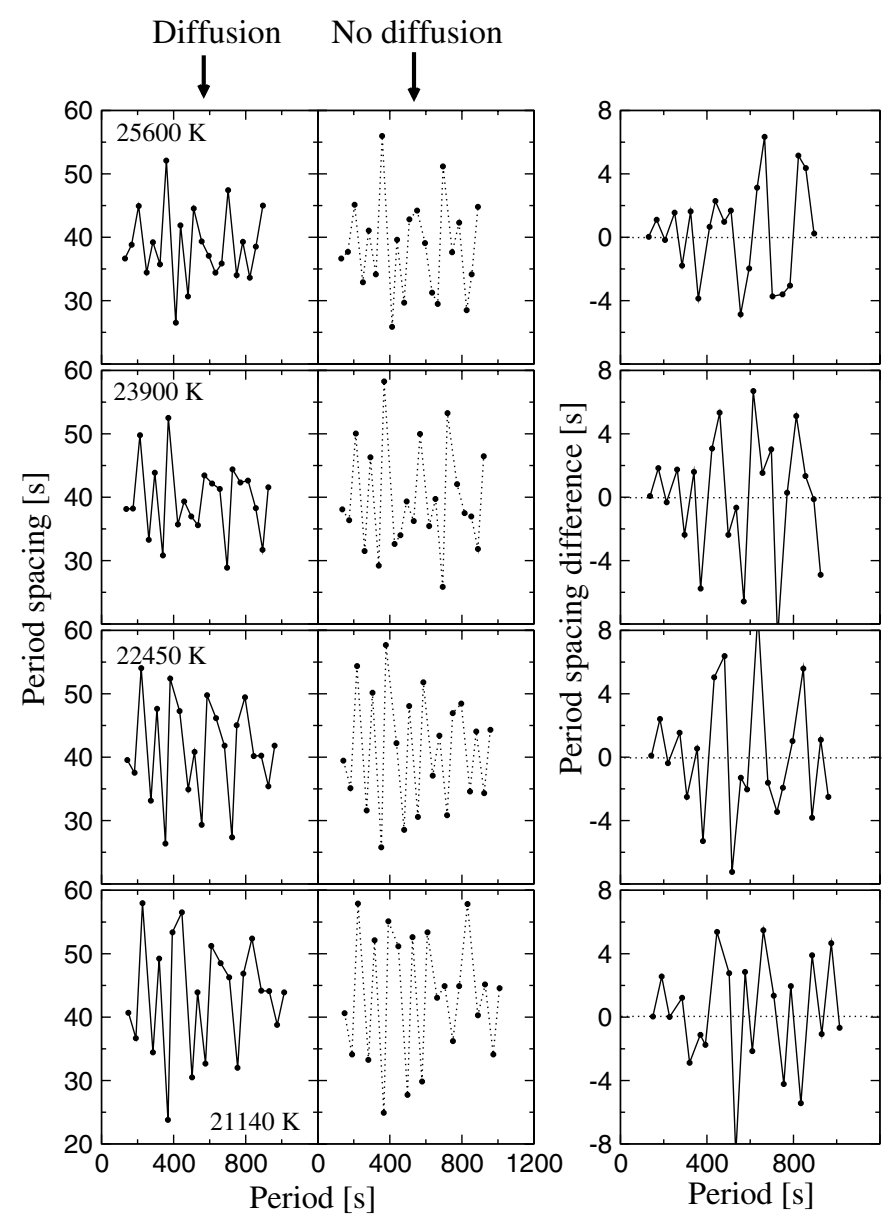

Fig. 9. Period spacing diagrams (for $\ell=1$ modes) for the case of 0.6- $M_{\odot}$ WD models with a helium content of $M_{\mathrm{He}}=8 \times 10^{-3} M_{*}$. From top to bottom panels correspond to decreasing effective temperatures (labels in the left panels). The left (centre) column of panels shows the results obtained when element diffusion has (not) been considered in the computations. Right panels depict the differences between the period spacing as given by diffusively evolving profiles and a fixed one predicted by diffusion at $T_{\text {eff }} \approx 27000 \mathrm{~K}$.

coupled if the helium mass is less massive enough for a singlelayered profile to emerge.

\section{Conclusions and discussion}

In this paper we have explored the evolution of white dwarf stars with helium-rich atmospheres (DB) in a self-consistent way with the predictions of time-dependent element diffusion. The focus is on the chemistry variations along the effective temperature range where these stars are found to pulsate. There exist theoretical and observational evidence (Dehner \& Kawaler 1995; Dreizler \& Heber 1998) suggesting that the majority of the DB white dwarfs could be the descendants of PG 1159 stars, the hot and hydrogen-deficient remnants of post-AGB stars that experienced a very late helium thermal pulse on their early cooling phase (see Herwig et al. 1999). In this regard, we have assumed for our starting DB white dwarf models an uniform envelope chemical composition rich in helium, carbon and oxygen. For this study, we have considered DB white dwarf models with stellar masses of 0.60 , 

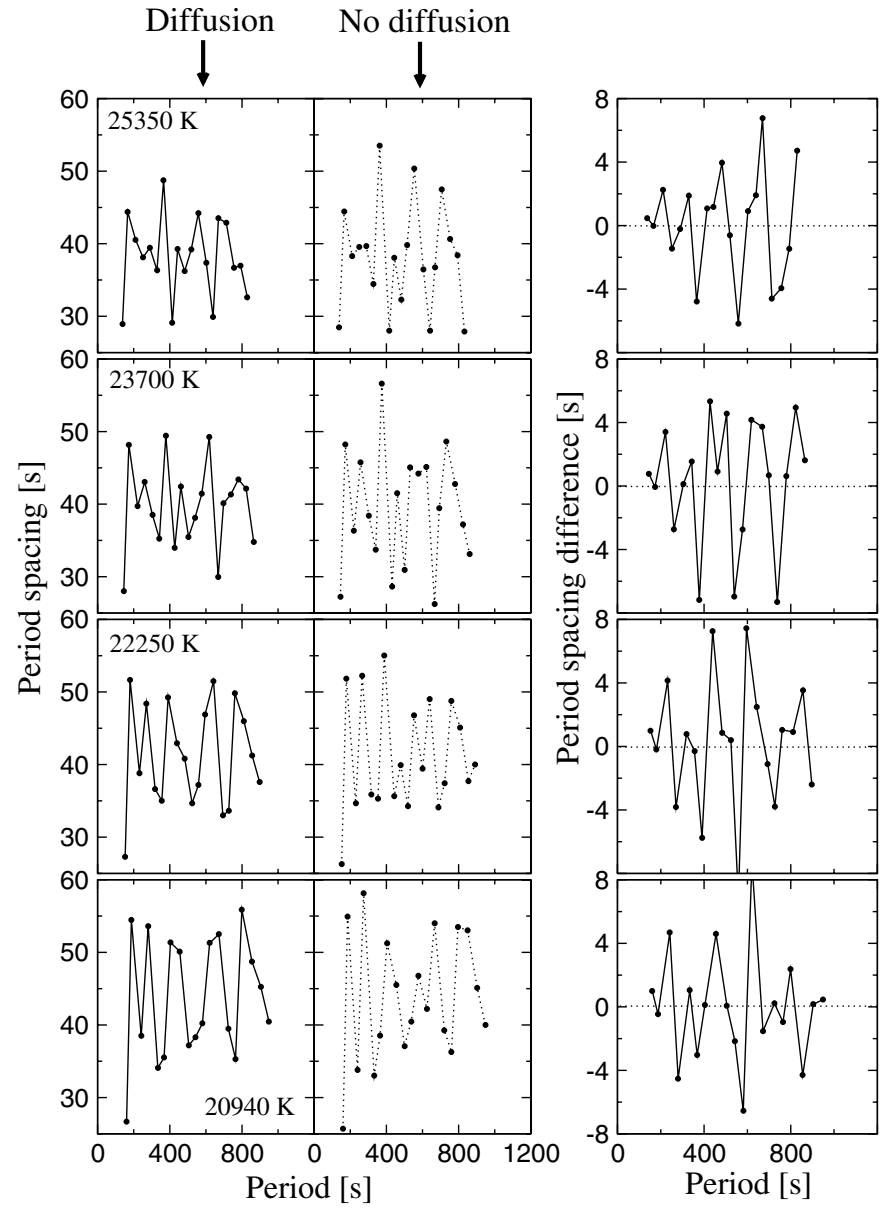

Fig. 10. Same as Fig. 9 but for a helium content of $M_{\mathrm{He}}=9 \times 10^{-4} M_{*}$.

$0.65,0.70,0.75$ and $0.85 M_{\odot}$. The mass of the helium envelope $\left(M_{\mathrm{He}}\right)$ was varied in the range $10^{-2}-10^{-4} M_{*}$. For a consistent treatment with the predictions of element diffusion, OPAL radiative opacities for arbitrary metallicity including carbonand oxygen-rich compositions have been employed. Our treatment of element diffusion, based on the multicomponent gas description of Burgers (1969), includes the effects of thermal diffusion, which, as we have shown, lead to DB models with thicker pure helium mantles.

In view of the lack of evolutionary calculations with time-dependent diffusion for DB white dwarfs with different stellar masses and helium envelopes, an initial question to be addressed in this work was whether the double-layered chemical profile expected for hot variable DBs (assuming that these stars descend from PG 1159 stars) could be markedly modified by further chemical evolution before the red edge of the instability strip is reached. This is an important point because as recently demonstrated by Fontaine \& Brassard (2002) the presence of a double-layered structure leads to a distinct theoretical period distribution from that predicted by single-layered configurations. Within the scope of the present paper is also the study of the consequences of diffusively evolving chemical profiles for the adiabatic pulsational properties of DB white dwarfs.

Our results show that, as found in previous studies, a double-layered envelope is expected by the time the white
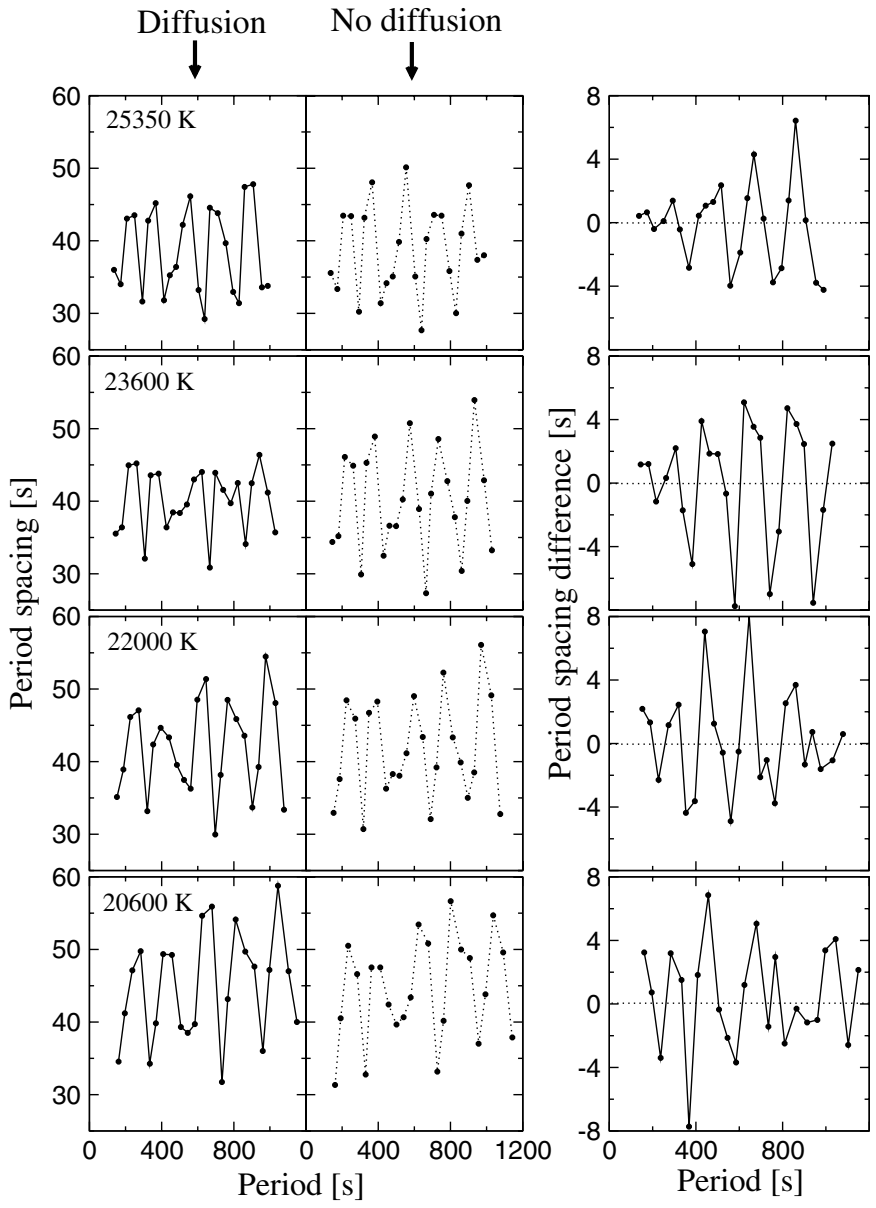

Fig. 11. Same as Fig. 9 but for a helium content of $M_{\mathrm{He}}=1 \times 10^{-4} M_{*}$.

dwarf reaches the domain of the DB pulsational instability. In particular, element diffusion not only thickens the pure helium mantle as cooling proceeds but also it modifies the shape of the chemical profile, an aspect to be considered in asteroseismological fittings of pulsating DB white dwarfs. In this regard, we find that the diffusively evolving shape of the envelope composition translates into a distinct behaviour of the theoretical period distribution as compared with the case in which the shape of the profile is assumed to be fixed during the DB white dwarf evolution across the instability strip. In particular, we find that the presence of a double-layered structure causes the period spacing diagrams to exhibit mode-trapping substructures which change due to diffusion.

Another finding of this work is the fact that the doublelayered structure is altered by further chemical evolution to such a degree that, depending on the helium content, a singlelayered configuration emerges even during the stages of pulsation instability. In particular, for a $0.6 M_{\odot}$ DB white dwarf with $M_{\mathrm{He}}=10^{-3} M_{*}$ this occurs by the time the star reaches the $T_{\text {eff }}$ value of $\approx 20000 \mathrm{~K}$, and for $M_{\mathrm{He}}=10^{-4} M_{*}$, a singlelayered configuration is expected at $T_{\mathrm{eff}} \approx 27000 \mathrm{~K}$. Thus, if post-AGB, DB white dwarf progenitors are formed with a helium content smaller than $\approx 10^{-3} M_{*}$, the double-layered structure is expected to become single-layered by the time the star reaches the red edge of the DB instability strip. A helium envelope mass of $\approx 10^{-3} M_{*}$ is more appropriate for a massive 
white dwarf. In particular, we find that the double-layered profile in a $\approx 0.8 M_{\odot}$ DB turns into a single-layered one well before the red edge of the DB instability domain is reached.

The existence of $0.6 M_{\odot}$ DB white dwarfs with a helium content less massive than, say, $\approx 10^{-3} M_{*}$ is difficult to reconcile with the predictions of the born-again scenario for the formation of hydrogen-deficient post-AGB stars. Indeed, Herwig et al. (1999) find that after the end of the late helium thermal pulse the total helium content left in the star amounts to $M_{\mathrm{He}} \approx$ $10^{-2} M_{*}$. Now, there exist observational evidence suggesting that post-AGB mass-loss episodes could reduce (and even remove) the mass of the helium-rich envelope considerably. As recently emphasized by Werner (2001) it is possible that the surface chemistry of all PG 1159 stars is determined not only by previous interior mixing processes but also by mass-loss episodes. Mass-loss rates in the range $10^{-7}-10^{-8} M_{\odot} / \mathrm{yr}$ are detectable in many luminous PG 1159 stars. In addition, tentative evidence for the persistence of mass-loss rates of the order $10^{-7}-10^{-10} M_{\odot} / \mathrm{yr}$ down to the domain of hot helium-rich white dwarfs has been presented (see Werner 2001). The existence of such mass-loss rates would imply that most of the helium-rich envelope of DB progenitors could be substantially reduced during the $10^{5}-10^{6} \mathrm{yr}$ elapsed during the post-AGB stage. It is worth mentioning that the existence of PG 1159 stars with a helium content as low as $10^{-3} M_{\odot}$ has been suggested by asteroseismology in at least one of these stars with a stellar mass of $0.6 M_{\odot}$ (Kawaler \& Bradley 1994), thus implying the occurrence of modest mass-loss during evolution to the PG 1159 phase. In light of such evidence, the possibility of the existence of some DB white dwarfs with helium contents lower than $10^{-3} M_{*}$ could not be discounted. According to our calculations, a double-layered structure would thus not be expected for such white dwarfs once they have reached the domain of DB pulsational instability.

Finally, we have extended the scope of our evolutionary calculations down to effective temperatures characteristics of the helium-rich carbon-contaminated DQ white dwarfs, the supposed cooler descendants of DBs. The presence of carbon in the surface of such stars is widely believed to result from convective dredge-up of the carbon diffusive tail by the superficial helium convection zone (Pelletier et al. 1986, see also Koester et al. 1982). New dredge-up carbon simulations in DQ stars by MacDonald et al. (1998) suggest that the low carbon abundance observed in many DQs could be explained in terms of a dredge-up effect only if such white dwarfs are characterized by very thick helium envelopes. As our results shown, if we assume that DQ white dwarfs may be linked to the post-AGB PG 1159 stars via the PG 1159-DB-DQ connection, then a $0.6 M_{\odot}$ DQ white dwarf with a helium content more massive than $10^{-2} M_{*}$ would be characterized by a double-layered structure. In particular, we find that the pure helium mantle extends down only to a fractional mass depth $q \approx 5 \times 10^{-5} M_{*}$ at $T_{\text {eff }}=10000 \mathrm{~K}$, shallow enough for carbon to be dredged-up to the surface with abundances far exceeding the observational predictions. If canonical convective dredge-up theory is correct, our results seem to discount an evolutionary connection between some DQs, those having low carbon surface abundances, and the PG 1159 stars. Full evolutionary calculations taking into account the evolutionary stages leading to the white dwarf formation, and particularly the chemical structure emerging from the born-again scenario, are required to place these assertions on a more solid basis. Work in this direction is in progress.

Detailed tabulations of the chemical profiles of our models are available at

http://www.fcaglp.unlp.edu.ar/evolgroup/

Acknowledgements. We warmly acknowledge Klaus Werner for sending us reprints central to this work. It is a pleasure to acknowledge our referee, whose comments and suggestions strongly improve the original version of this paper. This research was supported by the Instituto de Astrofísica La Plata.

\section{References}

Althaus, L. G., Serenelli, A. M., Córsico, A. H., \& Benvenuto, O. G. 2002, MNRAS, 330, 685

Bradley, P. A., \& Winget, D. E. 2001, ApJ, 430, 850

Brassard, P., Fontaine, G., Wesemael, F., Kawaler, S. D., \& Tassoul, M. 1991, ApJ, 367, 601

Burgers, J. M. 1969, Flow Equations for Composite Gases (New York: Academic)

Córsico, A. H., Althaus, L. G., Benvenuto, O. G., \& Serenelli, A. M. 2001, A\&A, 380, L17

Dehner, B. T., \& Kawaler, S. D. 1995, ApJ, 445, L141

Dreizler, S., \& Heber, U. 1998, A\&A, 334, 618

Fontaine, G., \& Brassard, P. 2002, ApJ, 581, L33

Gautschy, A., \& Althaus, L. G. 2002, A\&A, 382, 141

Heber, U. 1986, A\&A, 155, 33

Herwig, F., Blöcker, T., Langer, N., \& Driebe, T. 1999, A\&A, 349, L5

Iben, I. Jr., Kaler, J. B., Truran, J. W., \& Renzini, A. 1983, ApJ, 264, 605

Iglesias, C. A., \& Rogers, F. 1996, ApJ, 464, 943

Kawaler, S. D., \& Bradley, P. A. 1994, ApJ, 427, 415

Koester, D., Weidemann, V., \& Zeidler-K.T., E. M. 1982, A\&A, 116, 147

MacDonald, J., Hernanz, M., \& José, J. 1998, MNRAS, 296, 523

Magni, G., \& Mazzitelli, I. 1979, A\&A, 72, 134

Metcalfe, T. S., Montgomery, M. H., \& Kawaler, S. D. 2003, MNRAS, 344, L88

Metcalfe, T. S., Nather, R. E., \& Winget, D. E. 2000, ApJ, 545, 974

Metcalfe, T. S., Salaris, M. E., \& Winget, D. E. 2002, ApJ, 573, 803

Metcalfe, T. S., Winget, D. E., \& Charbonneau, P. 2001, ApJ, 557, 1021

Montgomery, M. H., Metcalfe, T. S., \& Winget, D. E. 2001, ApJ, 548, L53

Paquette, C., Pelletier, C., Fontaine, G., \& Michaud, G. 1986, ApJS, 61, 177

Pelletier, C., Fontaine, G., Wesemael, F., Michaud, G., \& Wegner, G. 1986, ApJ, 307, 242

Salaris, M., Domínguez, I., García-Berro, E., et al. 1997, ApJ, 486, 413

Tassoul, M., Fontaine, G., \& Winget, D. E. 1990, ApJS, 72, 335

Werner, K. 2001, Ap\&SS, 275, 27 